\title{
MUSCL discretization for the fluid flow convection operator on staggered meshes
}

A. Brunel ${ }^{1,2}$, R. Herbin ${ }^{1}$, J.-C. Latché ${ }^{2}$

\begin{abstract}
We propose in this paper a second order discretization of the momentum convection operator for fluid flow simulation on staggered quadrangular or hexahedral meshes. The velocity is approximated by the Rannacher-Turek finite element. The implemented MUSCL-like approach is of algebraic type, in the sense that the limitation procedure does not invoke any slope reconstruction, and is independent from the geometry of the cells. The derived discrete convection operator applies both to constant or variable density flows; we perform here numerical tests for the barotropic and incompressible Navier-Stokes equations.
\end{abstract}

Key words: Convection operator, fluid flow, MUSCL, staggered grid MSC (2010): 65M08, 76M12

\section{Introduction}

Several works combining a finite element approximation of diffusion terms with a finite volume discretization for the convection operator may be found in the literature. The implementation of such a technique to obtain monotone schemes for convection-diffusion equations may be found for instance in $[1,2,3]$. Since finite-volume convection operators (with suitable upwinding) also enjoy desirable $L^{2}$-stability properties, they have been used for the discretization of the NavierStokes equations, preferably for compatible accuracy, i.e. low order, approximations. An application of this strategy for the discretization of stationary incompressible Navier-Stokes equations by Crouzeix-Raviart finite elements may be found in [4]; extension to quasi-incompressible unsteady flows, both with the Crouzeix-

1 Aix Marseille University, CNRS, France. e-mail: (aubin.brunel, raphaele.herbin)@univ-amu.fr 2 Institut de Sûreté et de Radioprotection Nucléaire (IRSN), France. e-mail: (aubin.brunel, jeanclaude.latche)@irsn.fr 
Raviart and Rannacher-Turek finite elements, is performed in [5]. These two works only consider a first order upwinding technique, and our aim in this paper is to develop a second order convection operator, based on an algebraic MUSCL-like technique [6]. The obtained operator is quite general, in the sense that it may be applied to incompressible constant or variable density flows as well as to compressible flows, either for Navier-Stokes or Euler equations. We show here some numerical applications for the barotropic and the incompressible Navier-Stokes equations.

The continuous momentum convection operator that we consider here takes the following generic form $\partial_{t}(\rho \boldsymbol{u})+\operatorname{div}(\rho \boldsymbol{u} \otimes \boldsymbol{u})$ where $\rho$ is the fluid density and $\boldsymbol{u}$ the velocity. It may be recast under the form of a transport operator (which is central for its stability) provided that a mass balance equation holds, that is

$$
\partial_{t} \rho+\operatorname{div}(\rho \boldsymbol{u})=0,
$$

which we suppose here. The problem in which the convection operator is involved is supposed to be posed over $\Omega \times\left[0, T\right.$ ) where $\Omega \subset \mathbb{R}^{d}$ (with $d=2,3$ ) is an open bounded domain of boundary $\partial \Omega$ and $[0, T)$ is a finite time interval.

\section{Space and time discretizations}

We first define a primal mesh $\mathcal{M}$ by splitting $\Omega$ into a finite family of disjoint quadrangles (if $d=2$ ) or hexahedra (if $d=3$ ) denoted by $K$ and called control volumes or cells. We then denote by $\mathcal{E}$ the set of faces of the mesh $\mathcal{M}$; for $K \in \mathcal{M}, \mathcal{E}(K)$ stands for the set of faces of $K$ and we thus have $\partial K=\cup_{\sigma \in \mathcal{E}(K)} \sigma$. Any face $\sigma \in \mathcal{E}$ is either a part of the boundary of $\Omega$, i.e. $\sigma \subset \partial \Omega$, in which case $\sigma$ is said to be an external face, or there exists $(K, L) \in \mathcal{M}^{2}$ with $K \neq L$ such that $\bar{K} \cap \bar{L}=\sigma$ : we denote in this case $\sigma=K \mid L$ and $\sigma$ is said to be an internal face. We denote by $\varepsilon_{\text {ext }}$ and $\mathcal{E}_{\text {int }}$ the set of external and internal faces. For $K \in \mathcal{M}$ and $\sigma \in \mathcal{E}$, we denote by $|K|$ the measure of $K$ and $|\sigma|$ the $(d-1)$-measure of the face $\sigma$.

The discretization is staggered in the sense that the scalar and vector unknowns are not colocated. Indeed, the pressure and density unknowns are associated with the cells of the primal mesh $\mathcal{M}$ and denoted by $p_{K}, \rho_{K}$ while the degrees of freedom for the velocity are defined on a dual mesh using the Rannacher-Turek non-conforming low-order finite element approximation [7] and are denoted $\boldsymbol{u}_{\sigma}=\left(u_{\sigma, 1}, \ldots, u_{\sigma, d}\right)$. The dual mesh is constructed as follows: if $K \in \mathcal{M}$ is a rectangle or a rectangular cuboid, we denote by $x_{K}$ the mass center of $K$ and we construct $D_{K, \sigma}$ as the cone with basis $\sigma$ and with vertex $x_{K}$; this definition is extended to a general cell $K$, by supposing that $K$ is split in the same number of sub-cells (the geometry of which does not need to be specified) and with the same connectivity. We now define $D_{\sigma}$, the dual cell of basis $\sigma$, as $D_{\sigma}=D_{K, \sigma} \cup D_{L, \sigma}$ if $\sigma=K \mid L \in \mathcal{E}_{\text {int }}$ and $D_{\sigma}=D_{K, \sigma}$ if $\sigma \in$ $\varepsilon(K) \cap \varepsilon_{\text {ext }}$; its measure is denoted by $\left|D_{\sigma}\right|$. We then denote by $\tilde{\varepsilon}\left(D_{\sigma}\right)$ the set of dual faces of $D_{\sigma}$, and by $\varepsilon=D_{\sigma} \mid D_{\sigma^{\prime}}$ the face separating two dual cells $D_{\sigma}$ and $D_{\sigma^{\prime}}$. All the components of the velocity are then approximated on each face of the mesh, and 
their degrees of freedom are identified to the mean value of the velocity component over the face. An example of the discretization with a few control volumes is given on Figure 1.

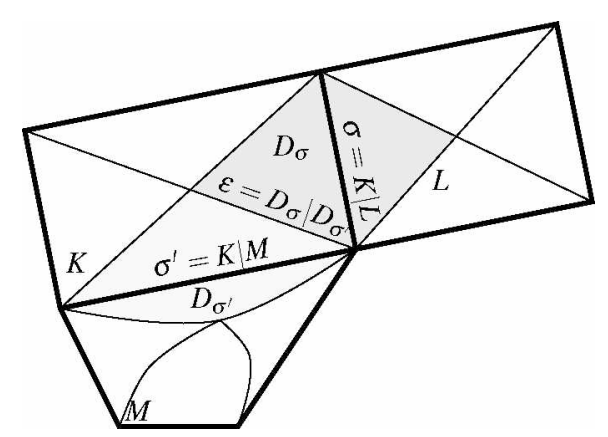

Fig. 1 Primal and dual meshes for the Rannacher-Turek elements.

Finally, a constant time step denoted by $\delta t$ is used for the time discretization, and for $0 \leq n \leq N=T / \delta t$, we define $t^{n}=n \delta t$.

\section{A second order discrete convection operator}

Let us first address the discretization of the mass balance equation (1) over the primal mesh. Mimicking the divergence theorem, the discrete divergence reads (dropping the time exponents for short), for $K \in \mathcal{M}$ :

$$
\operatorname{div}(\rho \boldsymbol{u})_{K}=\frac{1}{|K|} \sum_{\sigma \in \mathcal{E}(K)} F_{K, \sigma}
$$

where $F_{K, \sigma}$ stands for the (primal) numerical mass flux across $\sigma$ outward $K$ and is defined by:

$$
\forall \sigma=K\left|L \in \mathcal{E}_{\text {int }}, \quad F_{K, \sigma}=\right| \sigma \mid \rho_{\sigma} \boldsymbol{u}_{\sigma} \cdot \boldsymbol{n}_{K, \sigma}
$$

with $n_{K, \sigma}$ the normal vector to the face $\sigma$ outward $K$.

The dual mass fluxes $F_{\sigma, \varepsilon}$ for $\sigma \in \mathcal{E}$ and $\varepsilon \in \tilde{\varepsilon}\left(D_{\sigma}\right), \varepsilon \subset K$ are constructed from these primal fluxes so as to ensure that a discrete mass balance holds over the dual mesh $D_{\sigma}$; this is obtained by computing the face densities $\rho_{D_{\sigma}}$ and the mass fluxes $F_{\sigma, \varepsilon}$ as a linear combination of the densities in the primal cells adjacent to $\sigma$ and the mass fluxes through the primal faces of $K$, respectively; we refer to [8] for the exact expressions. 
These dual fluxes are then used for the definition of the discrete momentum convection operator, i.e. the discretization of the continuous term $\operatorname{div}\left(\rho u_{i} \boldsymbol{u}\right)$. For $1 \leq i \leq d$ and $\sigma \in \mathcal{E}$, the term $\operatorname{div}\left(\rho u_{i} \boldsymbol{u}\right)_{\sigma}$ reads:

$$
\operatorname{div}\left(\rho u_{i} u\right)_{\sigma}=\frac{1}{\left|D_{\sigma}\right|} \sum_{\varepsilon \in \tilde{E}\left(D_{\sigma}\right)} F_{\sigma, \varepsilon} u_{\varepsilon, i}
$$

where $u_{\varepsilon, i}$ is an approximation of $u_{i}$ over the face $\varepsilon$; it is obtained by the algebraic MUSCL-like technique introduced in [6], which implements the following procedure. Let us consider the explicit part of the convection term

$$
T_{\sigma, i}=\rho_{D_{\sigma}}^{n} u_{\sigma, i}^{n}-\delta t \operatorname{div}\left(\rho^{n} u_{i}^{n} \boldsymbol{u}^{n}\right)_{\sigma}
$$

The discrete convection operator is said to be monotone if the term $T_{\sigma, i}$ can be written as a convex combination of degrees of freedom of $u_{i}^{n}$; for instance, such a property would ensure a discrete maximum principle for the transport equation, or a convection-diffusion equation with a suitable (only available on specific meshes) discretization of the diffusion term. Such a formulation of the term $T_{\sigma, i}$ is possible by using the discrete mass balance over $D_{\sigma}$ if the following condition holds for each $\varepsilon \in \tilde{\varepsilon}_{\text {int }}$ such as $\varepsilon=D_{\sigma} \mid D_{\sigma^{\prime}}$ :

$$
\exists \alpha_{\varepsilon}^{\sigma} \in[0,1], \exists \tilde{\sigma} \in \mathcal{E} \text { such that } u_{\varepsilon, i}-u_{\sigma, i}=\mid \begin{aligned}
& \alpha_{\varepsilon}^{\sigma}\left(u_{\sigma, i}-u_{\tilde{\sigma}, i}\right) \text { if } F_{\sigma, \varepsilon} \geq 0, \\
& \alpha_{\varepsilon}^{\sigma}\left(u_{\tilde{\sigma}, i}-u_{\sigma, i}\right) \text { otherwise, }
\end{aligned}
$$

together with the following CFL condition:

$$
\mathrm{CFL}=\max _{\sigma \in \mathcal{E}}\left\{\frac{\delta t}{\left|D_{\sigma}\right|} \sum_{\varepsilon \in \tilde{\varepsilon}\left(D_{\sigma}\right)}\left|F_{\sigma, \varepsilon}\right|\right\} \leq 1
$$

We now deduce from the relation (5) a constructive process to compute the quantities $u_{\varepsilon, i}$. Let $\varepsilon$ be an internal face separating an upwind dual cell $D_{\sigma^{-}}$from the downstream dual cell $D_{\sigma^{+}}$(i.e. $\left.F_{\sigma^{-}, \varepsilon} \geq 0\right)$. Let us now choose two sets $\mathcal{N}_{\varepsilon}\left(D_{\sigma^{-}}\right)$ and $\mathcal{N}_{\mathcal{E}}\left(D_{\sigma^{+}}\right)$of neighbouring dual cells of $D_{\sigma^{-}}$and $D_{\sigma^{+}}$respectively. The following assumptions are then a transcription of Condition (5):

$$
\begin{aligned}
& \exists D_{\bar{\sigma}} \in \mathcal{N}_{\mathcal{\varepsilon}}\left(D_{\sigma^{+}}\right) \text {such that } u_{\mathcal{\varepsilon}, i} \in I_{1}=\left[u_{\bar{\sigma}, i}, u_{\bar{\sigma}, i}+\frac{\xi^{+}}{2}\left(u_{\sigma^{+}, i}-u_{\bar{\sigma}, i}\right)\right] ; \\
& \exists D_{\bar{\sigma}} \in \mathcal{N}_{\mathcal{\varepsilon}}\left(D_{\sigma^{-}}\right) \text {such that } u_{\mathcal{E}, i} \in I_{2}=\left[u_{\sigma^{-}, i}, u_{\sigma^{-}, i}+\frac{\xi^{-}}{2}\left(u_{\sigma^{-}, i}-u_{\bar{\sigma}, i}\right)\right]
\end{aligned}
$$

where $\xi^{+}$and $\xi^{-}$are two numerical parameters lying in the interval $[0,2]$.

Here we choose $\mathcal{N}_{\varepsilon}\left(D_{\sigma^{+}}\right)=\left\{D_{\sigma^{-}}\right\}$. Concerning $\mathcal{N}_{\varepsilon}\left(D_{\sigma^{-}}\right)$, several choices are possible; we choose here the opposite cell to $D_{\sigma^{+}}$with regard to $D_{\sigma^{-}}$, which means that if $D_{\sigma^{-}}$and $D_{\sigma^{+}}$share the primal face $\varepsilon$, we choose the cell $D_{\sigma^{\prime}}$ such that $D_{\sigma^{\prime}}$ shares a face $\varepsilon^{\prime}$ with $D_{\sigma^{-}}$and $\varepsilon$ and $\varepsilon^{\prime}$ share no vertex. With these choices, the 
upwind value is always admissible, and in fact it is the only admissible cell if the two parameters are chosen equal to 0 .

We are now in position to give the algorithm used to compute the quantities $u_{\varepsilon, i}$ :

1. Compute a tentative value $\bar{u}_{\varepsilon, i}$ with a convex combination of the values (e.g. the centered choice) in the surrounding faces.

2. Evaluate $F_{\sigma, \varepsilon}$ to determine the upwind face $D_{\sigma^{-}}$and the downwind face $D_{\sigma^{+}}$, and choose accordingly the neighbouring sets $\mathcal{N}_{\varepsilon}\left(D_{\sigma^{-}}\right)$and $\mathcal{N}_{\varepsilon}\left(D_{\sigma^{+}}\right)$.

3. Compute an admissible interval $I_{1} \cap I_{2}$ for $u_{\varepsilon, i}$ by (7).

4. Compute $u_{\varepsilon, i}$ by projecting the tentative value $\bar{u}_{\varepsilon, i}$ into the interval obtained in the previous step.

Since this procedure is not linear, we cannot expect to derive an explicit formula to compute the values of the coefficients $a_{\varepsilon}^{\sigma}$. Their evaluation is however useless for an explicit scheme: indeed,the presented algorithm univocally defines the value $u_{\varepsilon, i}$. However, for this reason, we cannot easily define an implicit-in-time MUSCL scheme (this would require an iterative process for each time step).

\section{Numerical tests}

The computations presented here are performed with the open-source $\mathrm{CALIF}^{3} \mathrm{~S}$ software developed at IRSN [9].

\subsection{Compressible Navier-Stokes equations}

We first show an application to the barotropic compressible case:

$$
\begin{aligned}
& \partial_{t}\left(\rho u_{i}\right)+\operatorname{div}\left(\rho u_{i} \boldsymbol{u}\right)+\partial_{i} p-\operatorname{div}\left(\mu\left(\nabla \boldsymbol{u}+\nabla \boldsymbol{u}^{t}\right)\right)_{i}=0 \quad(i \in \llbracket 1, d \rrbracket) \\
& \partial_{t}(\rho)+\operatorname{div}(\rho \boldsymbol{u})=0 \\
& p=a \rho^{\gamma}, \quad a>0, \gamma \geq 1 .
\end{aligned}
$$

where $p$ stands for the pressure.

The scheme - A first-order forward Euler time-discretization of System (8) reads:

$$
\forall K \in \mathcal{M}, \quad \frac{1}{\delta t}\left(\rho_{K}^{n+1}-\rho_{K}^{n}\right)+\operatorname{div}\left(\rho^{n} u^{n}\right)_{K}=0,
$$

For $1 \leq i \leq d, \forall \sigma \in \mathcal{E}$,

$$
\begin{gathered}
\frac{1}{\delta t}\left(\rho_{D_{\sigma}}^{n+1} u_{\sigma, i}^{n+1}-\rho_{D_{\sigma}}^{n} u_{\sigma, i}^{n}\right)+\operatorname{div}\left(\rho^{n} u_{i}^{n} \boldsymbol{u}^{n}\right)_{\sigma} \\
+(\nabla p)_{\sigma, i}^{n}-\operatorname{div}\left(\boldsymbol{\mu}\left(\nabla \boldsymbol{u}^{n}+\nabla\left(\boldsymbol{u}^{n}\right)^{t}\right)\right)_{\sigma, i}=0, \\
\forall K \in \mathcal{M}, p_{K}^{n+1}=a\left(\rho_{K}^{n+1}\right)^{\gamma},
\end{gathered}
$$


with the previously described discrete convection terms and with the discrete pressure gradient and momentum diffusion term as given in [10]. Second order in time is obtained by using the Heun scheme, which reads

$$
\boldsymbol{W}^{n+\frac{1}{3}}=S\left(\boldsymbol{W}^{n}\right), \quad \boldsymbol{W}^{n+\frac{2}{3}}=S\left(\boldsymbol{W}^{n+\frac{1}{3}}\right), \quad \boldsymbol{W}^{n+1}=\frac{1}{2}\left(\boldsymbol{W}^{n}+\boldsymbol{W}^{n+\frac{2}{3}}\right),
$$

where $W^{n}=\left(\rho^{n}, u^{n}, p^{n}\right)$ is the vector of unknowns at step $n$ and $S\left(W^{n}\right)$ is obtained by one step of the forward Euler (9) The Heun scheme is used in the following numerical test.

Translated standing vortex - Here we assess the convergence rate of the proposed scheme on a test case built to this purpose. We first derive an analytical solution of the steady barotropic Euler equations consisting in a standing vortex; then this solution is made unsteady by adding a constant velocity translation. A solution for the Navier-Stokes equations is finally derived by compensating the viscous forces (that appear on the left hand side of equation (8a)) with a source term. We refer to [11] for the expression of this solution.

The viscosity $\mu$ is chosen so that the Reynolds number is equal to $50, a=9.81 / 2$ and $\gamma=2$. The domain is the square $\Omega=[-1.2,2]^{2}$ and the computation is run on the time interval $[0,0.8]$. Uniform $n \times n$ grids are used, starting from $n=32$ and doubling the number of control volumes in each direction up to $n=256$ mesh. The time step is set to $0.03125 \times h$, with $h=3.2 / n$, which yields a CFL number with respect to the celerity of the fastest wave close to 0.01 (the material velocity and the speed of sound are in the range of 1.45 and 0.76 respectively); this low value of the CFL number is imposed by the explicit discretization of the diffusion term (the constraint stems from the necessity to be stable up to the finest mesh). On Figure
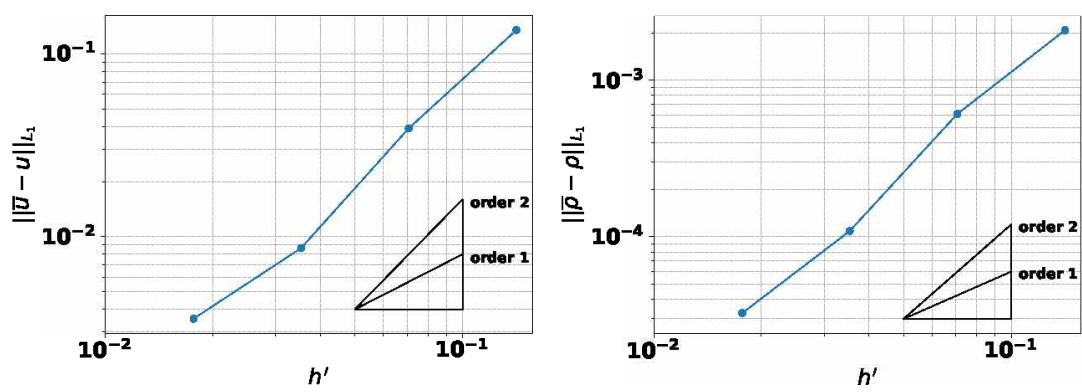

Fig. $2 L^{1}$ norm error for the MUSCL scheme for the velocity and the density. Here $h^{\prime}=$ $\max _{K \in \mathcal{M}} \operatorname{diam}(K)=\sqrt{2} h$.

2, we draw the $L^{1}$ norm of the numerical error for the velocity and the density as a function of the mesh step. This error is obtained by taking the difference between the computed velocity or density at the final time and the piecewise constant function defined by taking on each dual cell the value of the continuous solution at the cell 
center. The measured convergence orders are close to 1.8 and 2 for the velocity and the density respectively.

\subsection{Incompressible Navier-Stokes equation}

We now turn to the incompressible Navier-Stokes equations (with $\rho=1$ ):

$$
\begin{aligned}
& \partial_{t} u_{i}+\operatorname{div}\left(u_{i} \boldsymbol{u}\right)+\partial_{i} p-\operatorname{div}\left(\boldsymbol{\mu}\left(\boldsymbol{\nabla} \boldsymbol{u}+\nabla \boldsymbol{u}^{t}\right)\right)_{i}=0 \quad(i \in \llbracket 1, d \rrbracket) \\
& \operatorname{div}(\boldsymbol{u})=0
\end{aligned}
$$

The scheme - This system is solved using a projection scheme, which consists in the two following steps:

$$
\begin{aligned}
& \text { Prediction step - Solve for } \tilde{\boldsymbol{u}}^{n+1} \text { : } \\
& \begin{aligned}
\text { For } 1 \leq i \leq d, \forall \sigma \in \mathcal{E}, \quad & \frac{1}{\delta t}\left(\tilde{\boldsymbol{u}}_{\sigma, i}^{n+1}-\boldsymbol{u}_{\boldsymbol{\sigma}, i}^{n}\right)+\operatorname{div}\left(\tilde{\boldsymbol{u}}_{i}^{n} \boldsymbol{u}^{n}\right)_{\sigma} \\
& +(\boldsymbol{\nabla} p)_{\sigma, i}^{n}-\operatorname{div}\left(\mu\left(\nabla \tilde{\boldsymbol{u}}^{n+1}+\left(\boldsymbol{\nabla} \tilde{\boldsymbol{u}}^{n+1}\right)^{t}\right)\right)_{\sigma, i}=0 .
\end{aligned}
\end{aligned}
$$

Correction step - Solve for $p^{n+1}$ and $\boldsymbol{u}^{n+1}$ :

$$
\begin{aligned}
& \text { For } 1 \leq i \leq d, \forall \sigma \in \mathcal{E}, \quad \frac{1}{\delta}\left(\boldsymbol{u}_{\sigma, i}^{n+1}-\tilde{\boldsymbol{u}}_{\sigma, i}^{n+1}\right)+\left(\boldsymbol{\nabla} p^{n+1}\right)_{\sigma, i}-\left(\nabla p^{n}\right)_{\boldsymbol{\sigma}, i}=0, \\
& \forall K \in \mathcal{M}, \quad \operatorname{div}\left(\boldsymbol{u}^{n+1}\right)_{K}=0
\end{aligned}
$$

The convection terms are defined in the previous section, with the density set to 1 in the mass flux. We refer once again for short to [10] for the definition of the discrete pressure gradient and the momentum diffusion term.

Flow past a cylinder - We consider here a flow past a cylinder studied in a literature benchmark [12]. The geometry of the domain is given in [12, Figure 1]. The present test corresponds to the Test Case 2D-2 of [12]. The viscosity $\mu$ is chosen so that the Reynolds number is equal to 5000 , so the convection is strongly dominant. The computations are performed using a very coarse grid with 4033 cells, representative of what may be encountered in very complex 3D industrial simulations. We compare the results with the present convection scheme with the results obtained with (implicit-in-time) upwind and centered convection operators.

The main quantities of interest are the pressure difference $\Delta P$ between the front and end points of the cylinder, the Strouhal number, the maximum drag coefficient and the maximal and minimal lift coefficients. They are gathered in Table 1 and Table 2, together with reference values obtained with a converged-in-space computation. Even if the convergence is far from being reached with the (intentionally) very coarse mesh used in this study, the MUSCL scheme seems able to capture at least the order of magnitude of the reported quantities. This is supported by an examination of the computed flow structure: the vortex shedding phenomenon is qual- 
itatively reproduced by the MUSCL scheme while the upwind and centered ones yield, respectively, unrealistic small and large recirculation zones.

\begin{tabular}{l|c|c}
\multicolumn{3}{c}{ Reference } \\
$\Delta P_{\max }$ & $S_{t}$ \\
\hline 3.33080 & 0.3371 \\
Scheme & $\Delta P_{\max }$ & $S_{t}$ \\
\hline Upwind & 2.37020 & 0.2304 \\
Centered & 2.00400 & 0.2591 \\
MUSCL & 2.63470 & 0.2660
\end{tabular}

Table $1 \Delta P$ and Strouhal number.

Reference
\begin{tabular}{c|c|c|c|c}
$c_{d, \max }$ & $c_{l, \max }$ & $c_{l, \min }$ \\
\hline 3.46088 & 2.8479 & -2.78536 \\
Scheme & $c_{d, \max }$ & $c_{l, \max }$ & $c_{l, \min }$ \\
\hline Upwind & 3.23544 & 0.52157 & -0.51295 \\
Centered & 3.18728 & 0.10793 & -0.16129 \\
MUSCL & 3.42478 & 1.23162 & -1.16792
\end{tabular}

Table 2 Lift and drag coefficients.

\section{References}

1. K. Ohmori and T. Ushijima, "A technique of upstream type applied to a linear nonconforming finite element approximation of convective diffusion equations," RAIRO. Analyse numérique, vol. 18, pp. 309-332, 1984.

2. L. Angermann, "Numerical solution of second-order elliptic equations on plane domains," Mathematical Modelling and Numerical Analysis, vol. 25, pp. 169-191, 1991.

3. R. Eymard, D. Hilhorst, and M. Vohralík, "A combined finite volume-nonconforming/mixedhybrid finite element scheme for degenerate parabolic problems," Numerische Mathematik, vol. 105, pp. 73-131, 2006.

4. F. Schieweck and L. Tobiska, "An optimal order error estimate for an upwind discretization of the Navier-Stokes equations," Numerical Methods for Partial Differential Equations, vol. 12, pp. 407-421, 1996.

5. G. Ansanay-Alex, F. Babik, J. Latché, and D. Vola, "An L2-stable approximation of the Navier-Stokes convection operator for low-order non-conforming finite elements," International Joumal for Numerical Methods in Fluids, vol. 66, pp. 555-580, 2011.

6. L. Piar, F. Babik, R. Herbin, and J,-C. Latché, "A formally second-order cell centred scheme for convection-diffusion equations on general grids," International Journal for Numerical Methods in Fluids, vol. 71, pp. 873-890, 2013.

7. R. Rannacher and S. Turek, "Simple nonconforming quadrilateral Stokes element," Numerical Methods for Partial Differential Equations, vol. 8, pp. 97-111, 1992.

8. R. Herbin, J.-C. Latché, and T. Nguyen, "Consistent segregated staggered schemes with explicit steps for the isentropic and full Euler equations," Mathematical Modelling and Numerical Analysis, vol. 52, pp. 893-944, 2018.

9. CALIF ${ }^{3}$ S, "A software components library for the computation of fluid flows." https://gforge.irsn.fr/gf/project/califs.

10. D. Grapsas, R. Herbin, W. Kheriji, and J.-C. Latché, "An unconditionally stable staggered pressure correction scheme for the compressible Navier-Stokes equations," SMAI Journal of Computational Mathematics, vol. 2, pp. 51-97, 2016.

11. T. Gallouët, R. Herbin, J.-C. Latché, and Y. Nasseri, "A second order consistent MAC scheme for the shallow water equations," this conference, 2020.

12. M. Schäfer, S. Turek, F. Durst, E. Krause, and R. Rannacher, "Benchmark computations of laminar flow around a cylinder," in Flow simulation with high-performance computers II, pp. $547-566,1996$. 